\title{
CAMBIO DE FECHA DE SIEMBRA DEL MAÍZ EN CULIACÁN, SINALOA, MÉXICO
}

\author{
CHANGE IN MAIZE PLANTING DATE AT CULIACÁN, SINALOA, MÉXICO
}

\author{
José L. Ramírez Díaz ${ }^{1 *}$, J. Jesús Wong Pérez² ${ }^{2}$ José A. Ruiz Corral $^{1}$ y Margarito Chuela Bonaparte ${ }^{1}$
}

\begin{abstract}
${ }^{1}$ Campo Experimental Centro-Altos de Jalisco, Instituto Nacional de Investigaciones Forestales, Agrícolas y Pecuarias (INIFAP). km 8 Carr. Libre TepatitlánLagos de Moreno. 47600, Tepatitlán, Jal., México. Tel. y Fax (378)782-0355. ${ }^{2}$ Campo Experimental Valle de Culiacán, INIFAP. km 17.5 Carr. Culiacán-El Dorado. 80000, Culiacán, Sin. México.

* Autor para correspondencia (ramirez.joseluis@inifap.gob.mx)
\end{abstract}

\section{RESUMEN}

En el Estado de Sinaloa, México, son dinamicos los cambios en prácticas agrícolas, tipos de variedad y estrategias de mejoramiento genético del cultivo del maíz (Zea mays $\mathbf{L}$.), lo que ha permitido que el rendimiento de grano se incrementara en $63 \%$ durante el periodo 1991-1993. Al respecto, los agricultores han adelantado la fecha de siembra del ciclo otoño-invierno (O-I) en relación al periodo óptimo determinado en estudios previos. En el ciclo OI 2002-2003 se sembraron, en condiciones de riego, ocho híbridos comerciales en cinco fechas de siembra espaciadas cada 15 d, de noviembre 15 a enero 15. Se tomaron datos de rendimiento de grano, días a floración masculina y femenina, altura de planta y mazorca, porcentaje de acame de raíz y tallo, calificación de roya (Puccinia sorghi) y de mazorca, así como las temperaturas diarias máxima, mínima y media. Hubo diferencias $(P \leq 0.01)$ en rendimiento de grano entre fechas de siembra, híbridos y para la interacción fechas de siembra $\mathrm{x}$ híbridos. El rendimiento más alto (promedio de los ocho híbridos) se obtuvo en la siembra de noviembre $15,32 \mathrm{~d}$ antes que la fecha recomendada para el ciclo 1990-1991 (Diciembre 17). Los híbridos de grano blanco ' $\mathrm{H}-375$ ' y 'H-438' tuvieron el rendimiento de grano más alto (promedio de cinco fechas de siembra), y 'P-31G98 $Y$ ' el más bajo. Las diferencias en temperatura entre los ciclos agrícolas, especialmente la temperatura máxima diaria, fue el factor más importante para explicar la interacción fecha de siembra $x$ híbrido.

Palabras clave: Zea mays, adaptación, prácticas agrícolas.

\section{SUMMARY}

In the State of Sinaloa, México, the changes in agricultural practices, variety types, and breeding strategies in maize (Zea mays L.) have been very dynamic, which has allowed that grain yield was increased by $63 \%$ during the period 1991-2003. On this regard, farmers have advanced the planting date of the cycle AutumnWinter (A-W) in relation to the previously determined optimal period. In the cycle A-W 2002-2003 were planted, in irrigation, eight commercial hybrids in five planting dates, $15 \mathrm{~d}$ apart each, from November 15 to January 15. Data collected were grain yield, days to male and female flowering, plant and ear height, percentage of root and stalk lodging, visual rate of rust (Puccinia sorghi) and ear, as well as the daily maximum, minimum and average temperatures. There were differences $(P \leq 0.01)$ in grain yield among planting dates, hybrids, and planting date $x$ hybrid interaction. The highest grain yield (average of the eight hybrids) was recorded in the plating date of November 15, $32 \mathrm{~d}$ before the planting date recommended for the cycle 1990-1991 (December 17). The white grain hybrids ' $H$ 375' and ' $\mathrm{H}-438$ ' showed the highest grain yield (average of five planting dates), while the lowest yield var in 'P-31G88 Y'. The differences in temperature between the agricultural cycles, specially in the daily maximum temperature, was the most important factor to explain the interaction planting date $\mathbf{x}$ hybrid.

Index words: Zea mays, adaptation, agricultural practices.

\section{INTRODUCCIÓN}

En México el maíz (Zea mays L.) se siembra en dos ciclos agrícolas: Primavera-Verano (PV) y OtoñoInvierno (OI); la mayor superficie y volumen de producción se obtiene en PV; sin embargo, en el noroeste del país, particularmente en el Estado de Sinaloa, el ciclo OI es más importante; $i$. e., en el ciclo 2005-2006 se cosecharon 420908 ha con un rendimiento promedio de $9.78 \mathrm{t} \mathrm{ha}^{-1}$ (SAGARPA, 2007). En este ciclo agrícola el maíz se cultiva principalmente en áreas con riego, lo cual permite al productor usar con mayor eficiencia los factores de la producción y obtener un alto rendimiento de grano. La época de siembra y la variedad son factores críticos en este sistema de producción. Las variedades responden diferencialmente a los factores del ambiente (principalmente, plagas, enfermedades y temperatura), que se manifiestan en diversos grados a lo largo de la estación del crecimiento y que impactan el rendimiento económico y la calidad del producto (Wiatrak et al., 2004, 2005; Barrios et al., 2004).

La temperatura es un factor importante de la fecha de siembra en el ciclo de OI; si se siembra demasiado temprano, las plantas sufren temperaturas bajas propias del invierno durante las primeras etapas de su desarrollo que les ocasiona retrasos en el crecimiento y desarrollo; si la siembra es muy tardía, las plantas estarán expuestas a 
altas temperaturas diurnas y nocturnas en la etapa de llenado de grano, que les aumenta sus tasas respiratorias y reduce su rendimiento de grano (Benacchio, 1982; Ojeda et al., 2006). El fotoperiodo no se considera un factor restrictivo en la estación de crecimiento de OI, pues el maíz es una planta de fotoperiodo corto o insensible a este factor (Francis, 1972); además, el número de horas luz se incrementa a partir del 20-22 de diciembre (solsticio de invierno) hasta llegar a su máximo el 21-22 de junio (solsticio de verano), después de la cosecha.

Medina (1993; Com. Personal ${ }^{1}$ ) evaluó dos variedades de maíz de polinización libre y cuatro híbridos en el Valle de Culiacán, Sin., en 14 fechas de siembra (septiembre 17 a abril 1); encontró que el rendimiento de grano se ajustó a una distribución polinomial de segundo orden, con un valor máximo en las fechas de diciembre 17 y enero 2 , y concluyó que el mejor periodo de siembra era de noviembre 14 a febrero 7. Sin embargo, 10 años después la producción de maíz en Sinaloa ha tenido cambios importantes, sobre todo en el tipo de variedades que se comercializan, la época de siembra y las prácticas de cultivo. En 1990 se utilizaban variedades de polinización libre y cruzas dobles (Medina, 1993; Opus cit.); actualmente predominan híbridos de cruza simple, de cruza simple modificada y cruzas trilineales, densidades de población altas y fechas de siembra adelantadas hacia noviembre. En consecuencia, se consideró necesario realizar un nuevo estudio de fechas de siembra con los actuales híbridos comerciales de maíz, y analizar las causas que han influido para que los productores hayan hecho tales cambios.

\section{MATERIALES Y MÉTODOS}

El presente estudio se efectuó durante el ciclo agrícola OI 2002-2003 en el Valle de Culiacán ( $24^{\circ} 48^{\prime} 28^{\prime}$ ' LN, $107^{\circ} 24^{\prime} 30^{\prime}$ ' LO, $38 \mathrm{~m}$ de altitud). Los tratamientos fueron cinco fechas de siembra y ocho híbridos. Las fechas de siembra fueron: noviembre 15 y 30 , diciembre 15 y 31 de 2002 y enero 15 de 2003, que incluyen el periodo de diciembre 17 a enero 2 en el cual Medina (1993; Opus cit.) obtuvo el mayor rendimiento. Los híbridos comerciales de maíz de grano blanco fueron: 'León', 'P-30G54', 'H-375' y 'H-438', y de grano amarillo: 'Potro Y', 'Pantera Y', 'Z-806', y 'P-31G98 Y'. Los híbridos 'León', 'Potro Y', 'Z-806' y 'Pantera Y' son trilineales; 'P-30G54', 'H-375', 'H-438' y 'P-

\footnotetext{
${ }^{1}$ Medina P G (1993) Respuesta de seis variedades de maíz (Zea mays L.) en catorce fechas de siembra en el Valle de Culiacán. Tesis de Licenciatura. Escuela Superior de Agricultura Fitotecnia. Depto. de Servicio Social. Universidad Autónoma de Sinaloa. Culiacán, Sin. México. 72 p.
}

31G98 Y' son simples. Los híbridos 'H-375', 'H-438', 'P-30G54', 'León' y 'Pantera Y' son de ciclo tardío; 'P31G98 Y', 'Z-806 Y' y 'Potro Y' son de ciclo intermedio-tardío. No se incluyeron híbridos más precoces porque la estación de crecimiento en Culiacán es mayor de $180 \mathrm{~d}$ y en condiciones de riego la meta del productor es obtener el máximo rendimiento de grano, lo cual no sería posible con un híbrido más precoz.

Las fechas de siembra se analizaron como experimentos en serie (Martínez, 1996), donde cada experimento fue una fecha de siembra con los ocho híbridos. El diseño experimental en cada fecha fue bloques completos al azar con cuatro repeticiones; la unidad experimental fue de dos surcos de $5.0 \mathrm{~m}$ de longitud espaciados a $0.80 \mathrm{~m}$ con 25 plantas cada uno (62 500 plantas ha $^{-1}$ ). El suelo del lote experimental es de textura arcillosa con $\mathrm{pH}$ de 7.5 a $7.6 \mathrm{y}$ materia orgánica $<1.0 \%$. El manejo agronómico de experimentos fue igual en todos, y consistió en un riego de presiembra y cuatro posteriores; fertilización con la fórmula $350 \mathrm{~N}-50 \mathrm{P}-00 \mathrm{~K}$ aplicada en dos oportunidades: tres cuartas partes del $\mathrm{N}$ y todo el $\mathrm{P}_{2} \mathrm{O}_{5}$ a la siembra, y el resto del $\mathrm{N}$ antes del primer riego de auxilio. La maleza se controló con el herbicida Acetocloro ${ }^{\circledR}$ aplicado en preemergencia $\left(2.0 \mathrm{~L} \mathrm{ha}^{-1}\right)$, las plagas del follaje (gusano cogollero Spodoptera frugiperda J. E. Smith, trips Frankliniella spp. y pulga saltona Epitix spp.), se controlaron con tres aplicaciones del insecticida Spinosad ${ }^{\circledR}(0.3$ $\left.\mathrm{L} \mathrm{ha}^{-1}\right)$.

Se midieron las siguientes variables: rendimiento de grano a $14 \%$ de humedad $\left(\mathrm{kg} \mathrm{ha}^{-1}\right)$; días a floración masculina (desde la siembra hasta que las espigas de $50 \%$ de las plantas de la parcela estuvieron liberando polen), días a floración femenina (cuando $50 \%$ de plantas tenían estigmas expuestos mayores que $5 \mathrm{~cm}$ ); altura de planta y mazorca $(\mathrm{cm})$, número de mazorcas/planta; porcentaje de acame de raíz y tallo; calificación del ataque de roya (Puccinia sorghi), donde 1 = follaje totalmente dañado y $9=$ sin daño; y calificación de aspecto de mazorca, donde 1 = mazorcas mal formadas, dañadas o podridas y 9 = mazorcas bien formadas y sin daño.

Se hicieron los siguientes análisis (SAS Institute, 1996): a) Análisis de varianza combinado, de todas las variables, con las cinco fechas de siembra; b) Análisis de correlación entre la fecha de siembra y el rendimiento de grano, calificación de roya, aspecto de mazorca y número de mazorcas/planta; c) Análisis de regresión del rendimiento de grano y de la floración femenina con la fecha de siembra; y d) Análisis de regresión del rendimiento de grano de cada híbrido con la fecha de siembra. Los datos de porcentaje, acame de raíz y tallo, se transformaron 
previamente con la función $\sqrt{\mathrm{X}}$ (Snedecor y Cochran, 1974).

La base de datos climáticos del Instituto Nacional de Investigaciones Forestales, Agrícolas y Pecuarias (INIFAP) (Ruiz et al., 2003; González et al., 2005) y de su Campo Experimental Valle de Culiacán se utilizaron para aplicar un análisis de correlación entre las temperatura media, máxima y mínima diarias históricas (19812003) del periodo de octubre a junio vs. las correspondientes a los ciclos OI 1990-1991 estudiado por Medina (1993; Opus cit.) y 2002-2003 del presente estudio, para conocer el grado de similitud de la temperatura diaria.

\section{RESULTADOS Y DISCUSIÓN}

En el análisis de varianza combinado (datos no presentados), las fechas de siembra e híbridos tuvieron efectos significativos $(\mathrm{P} \leq 0.01)$ en todas la variables, excepto en el porcentaje de acame de tallo del factor híbridos. La interacción fecha $\mathrm{x}$ híbridos fue significativa $(\mathrm{P} \leq 0.05)$ para rendimiento de grano, floración masculina, porcentaje de acame de raíz y tallo y aspecto de mazorca; es decir, los híbridos tuvieron un comportamiento diferencial al cambiar la fecha de siembra, lo cual coincide con lo informado por Barrios et al. (2004) y Wiatrak et al. (2004, 2005).

En promedio de los híbridos, el rendimiento de grano más alto se obtuvo en noviembre 15 (Cuadro 1), que disminuyó conforme la fecha de siembra se hizo más tarde (Figura 1). Esta tendencia se ajustó a un modelo de regresión lineal simple $\left(\mathrm{R}^{2}=0.96\right)$, e indica que por cada $15 \mathrm{~d}$ de retraso en la siembra el rendimiento se reduce en $748 \mathrm{~kg} \mathrm{ha}^{-1}$. Este resultado difiere de las fechas de máximo rendimiento (diciembre 17 y enero 2) señaladas por Medina (1993; Opus cit.) y apoyan la decisión de los productores de sembrar el maíz en el mes de noviembre.
Kucharik (2006), en la región Centro de los Estados Unidos, señala como posibles causas del adelanto en las fechas de siembra a la reducción del tiempo de preparación del suelo por el uso de labranza de conservación, la siembra de híbridos tardíos con mayor rendimiento y tolerancia a bajas temperaturas, la reducción del riesgo de heladas y daños por insectos y enfermedades, con menor énfasis en el cambio climático. En la primera fecha la incidencia de roya (Puccinia sorghi) fue menor y el número de mazorcas/planta fue mayor (Cuadro 1). La correlación entre la fecha de siembra y el rendimiento de grano fue negativa $(\mathrm{r}=-0.98)$ y significativa $(\mathrm{P} \leq 0.01)$. También las correlaciones entre fecha de siembra con incidencia de roya, aspecto de mazorca y número de mazorcas/planta fueron negativas $(-0.78,-0.78$ y -0.61 , respectivamente), pero no significativas.

La relación entre días a floración femenina (FF) y fecha de siembra, se ajustó a un modelo de regresión polinomial de segundo orden con valor máximo en la fecha del 15 de diciembre (Figura 1), la cual coincide con el valor máximo de floración femenina informado por Medina (1993; Opus cit.) y evidencia cierta similitud en las condiciones ambientales que prevalecieron en los ciclos agrícolas de 1990-1991 y de 2002-2003. Al respecto, el coeficiente de correlación entre la temperatura mínima y media diaria del periodo de octubre 1 a julio 31, entre los ciclos agrícolas de OI 1990-1991 (Medina, 1993; Opus cit.) y la del presente estudio (2002-2003), contra la media histórica (1981-2003) fueron similares, pero en la temperatura diaria máxima la correlación fue más alta en el ciclo 1990-1991 que en el ciclo 2002-2003 ( $\mathrm{r}=0.80 \mathrm{y}$ 0.77, respectivamente) (Cuadro 2). Asimismo, al analizar la similitud térmica entre esos dos ciclos agrícolas, el coeficiente de correlación más alto se obtuvo en la temperatura media diaria $(r=0.83)$, seguido por la mínima diaria $(\mathrm{r}=0.73)$, y el más bajo en la máxima diaria $(r=0.63)($ Cuadro 2). Es decir, las diferencias en

Cuadro 1. Rendimiento de grano y características agronómicas del maíz en cinco fechas de siembra (promedio de ocho híbridos). Culiacán, Sin. Ciclo Otoño-Invierno 2002-2003.

\begin{tabular}{|c|c|c|c|c|c|c|c|c|c|c|}
\hline \multirow{2}{*}{$\begin{array}{l}\text { Fecha de } \\
\text { Siembra (FS) }\end{array}$} & \multirow{2}{*}{$\begin{array}{c}\text { Rendimiento } \\
\left(\mathrm{kg} \mathrm{ha}^{-1}\right)\end{array}$} & \multirow{2}{*}{$\begin{array}{l}\text { Mazorcas } \\
\text { por planta }\end{array}$} & \multicolumn{2}{|c|}{ Floración (d) } & \multicolumn{2}{|c|}{ Altura $(\mathrm{cm})$} & \multicolumn{2}{|c|}{ Acame (\%) } & \multirow{2}{*}{$\begin{array}{l}\text { Calif. } \\
\text { roya }^{\dagger}\end{array}$} & \multirow{2}{*}{$\begin{array}{l}\text { Aspecto } \\
\text { mazorca }\end{array}$} \\
\hline & & & $\hat{0}$ & q & Planta & Mazorca & Raíz & Tallo & & \\
\hline Nov. 15 (FS1) & $11364 \mathrm{a}$ & $1.02 \mathrm{a}$ & $87 \mathrm{~d}$ & $87 \mathrm{~d}$ & $231 \mathrm{~b}$ & $129 \mathrm{a}$ & $3.2 \mathrm{a}$ & $0.72 \mathrm{a}$ & $9.0 \mathrm{a}$ & $8.4 \mathrm{ab}$ \\
\hline Nov. 30 (FS2) & $10853 \mathrm{~b}$ & $1.03 \mathrm{a}$ & $90 \mathrm{~b}$ & $90 \mathrm{~b}$ & $243 \mathrm{a}$ & $127 \mathrm{a}$ & $2.4 \mathrm{bc}$ & $0.22 \mathrm{~b}$ & $7.8 \mathrm{~b}$ & $8.4 \mathrm{ab}$ \\
\hline Dic. 15 (FS3) & $10223 \mathrm{c}$ & $1.01 \mathrm{a}$ & $91 \mathrm{a}$ & $91 \mathrm{a}$ & $223 \mathrm{bc}$ & $109 \mathrm{c}$ & $1.9 \mathrm{c}$ & $0.33 \mathrm{~b}$ & $7.6 \mathrm{~b}$ & $8.5 \mathrm{a}$ \\
\hline Dic. 31 (FS4) & $8906 \mathrm{~d}$ & $0.96 \mathrm{~b}$ & $88 \mathrm{c}$ & $88 \mathrm{c}$ & $215 \mathrm{c}$ & $112 \mathrm{c}$ & $2.2 \mathrm{c}$ & $0.19 \mathrm{~b}$ & $7.6 \mathrm{c}$ & $8.2 \mathrm{~b}$ \\
\hline Enero 15 (FS5) & $8596 \mathrm{~d}$ & $1.00 \mathrm{a}$ & $86 \mathrm{e}$ & $85 \mathrm{e}$ & $229 \mathrm{~b}$ & $118 \mathrm{~b}$ & $2.9 \mathrm{ab}$ & $0.11 \mathrm{~b}$ & $7.5 \mathrm{bc}$ & $7.6 \mathrm{c}$ \\
\hline DMS $_{0.05}$ & 332 & 0.03 & 0.61 & 0.64 & 9.0 & 5.6 & 0.6 & 0.32 & 0.4 & 0.25 \\
\hline
\end{tabular}




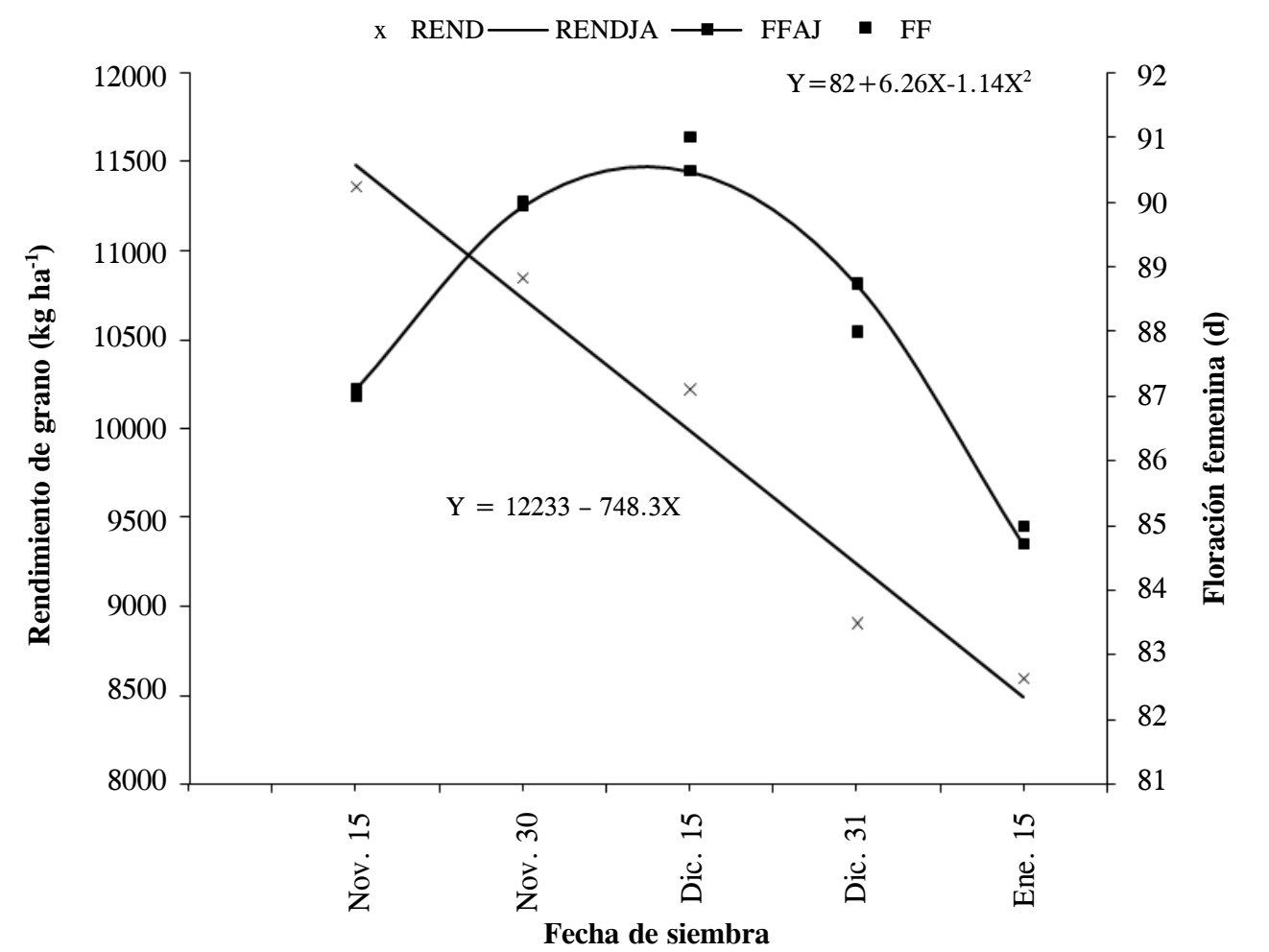

Figura 1. Rendimiento y floración femenina en función de la fecha de siembra (promedio de ocho híbridos). REND = Rendimiento sin ajustar; RENDJA = Rendimiento ajustado; FF = Fecha de floración femenina; FFAJ = Fecha de floración femenina ajustada. Culiacán, Sin. Ciclo Otoño-Invierno 2002-2003.

Cuadro 2. Correlación entre temperaturas media, máxima y mínima diaria de los ciclos agrícolas de Otoño-Invierno 1990-1991 y 2002-2003, y las del promedio histórico 1981 a 2003.

\begin{tabular}{llc}
\hline Temperatura diaria & & Coeficiente de correlación \\
\hline \multirow{2}{*}{ Máxima } & Histórica vs. Ciclo OI 1990-1991 & $0.80^{*}$ \\
& Histórica vs. ciclo OI 2002-2003 & $0.77^{*}$ \\
& Ciclo OI 1990-1991 vs. Ciclo OI 2002-2003 & $0.63^{*}$ \\
Mínima & Histórica vs. Ciclo OI 1990-1991 & $0.87^{*}$ \\
& Histórica vs. ciclo OI 2002-2003 & $0.88^{*}$ \\
& Ciclo OI 1990-1991 vs. Ciclo OI 2002-2003 & $0.73^{*}$ \\
Media & Histórica vs. Ciclo OI 1990-1991 & $0.92^{*}$ \\
& Histórica vs. ciclo OI 2002-2003 & $0.91^{*}$ \\
& Ciclo OI 1990-1991 vs. Ciclo OI 2002-2003 & $0.83^{*}$ \\
\hline
\end{tabular}

las fechas de máximo rendimiento de grano en los dos ciclos agrícolas podrían deberse, de manera conjunta, a la variación de la temperatura diaria, particularmente a la máxima, así como a los diferentes genotipos evaluados en cada estudio.

La distribución de las temperaturas máxima y mínima diarias del ciclo 2002-2003 (Figura 2) indica que en la siembra del 15 de noviembre (FS1), cuando se obtuvo el máximo rendimiento, las condiciones térmicas durante la emergencia de plántulas fueron más apropiadas que en las siembras del 30 de noviembre (FS2) y del 15 de diciembre (FS3), pues la temperatura mínima en FS1 fue superior a $10{ }^{\circ} \mathrm{C}$ (temperatura base) señalada como umbral para el desarrollo del maíz (Cross y Zuber, 1972; Shaw, 1975; Eskridge y Stevens, 1987). En contraste, en la FS2 y FS3 los genotipos estuvieron expuestos por más tiempo a temperatura superior $\left(30\right.$ a $\left.35{ }^{\circ} \mathrm{C}\right)$ a la del umbral considerado para el crecimiento del maíz (Cross y Zuber, 1972; Singh et al., 1976), lo cual ocasionó menor rendimiento de grano. En las siembras del 31 de diciembre y 15 de enero la temperatura no fue restrictiva 
para la emergencia, pero sí lo fue para el rendimiento de grano (Figuras 2 y 3 ).

En resumen, en el ciclo otoño-invierno de Culiacán, Sin., la temperatura mínima invernal alcanza valores inferiores a la temperatura base $\left(10^{\circ} \mathrm{C}\right)$, que reduce la velocidad de crecimiento y desarrollo del maíz sembrado en la primera quincena de diciembre; además, durante el llenado de grano inciden temperaturas diarias máximas por arriba de las temperaturas umbrales máximas, 30 a 35 ${ }^{\circ} \mathrm{C}$. Ojeda et al. (2006) determinaron que para el norte de Sinaloa, la mejor fecha de siembra de maíz es la primera quincena de octubre, lo cual coincide con la tendencia encontrada en este estudio.

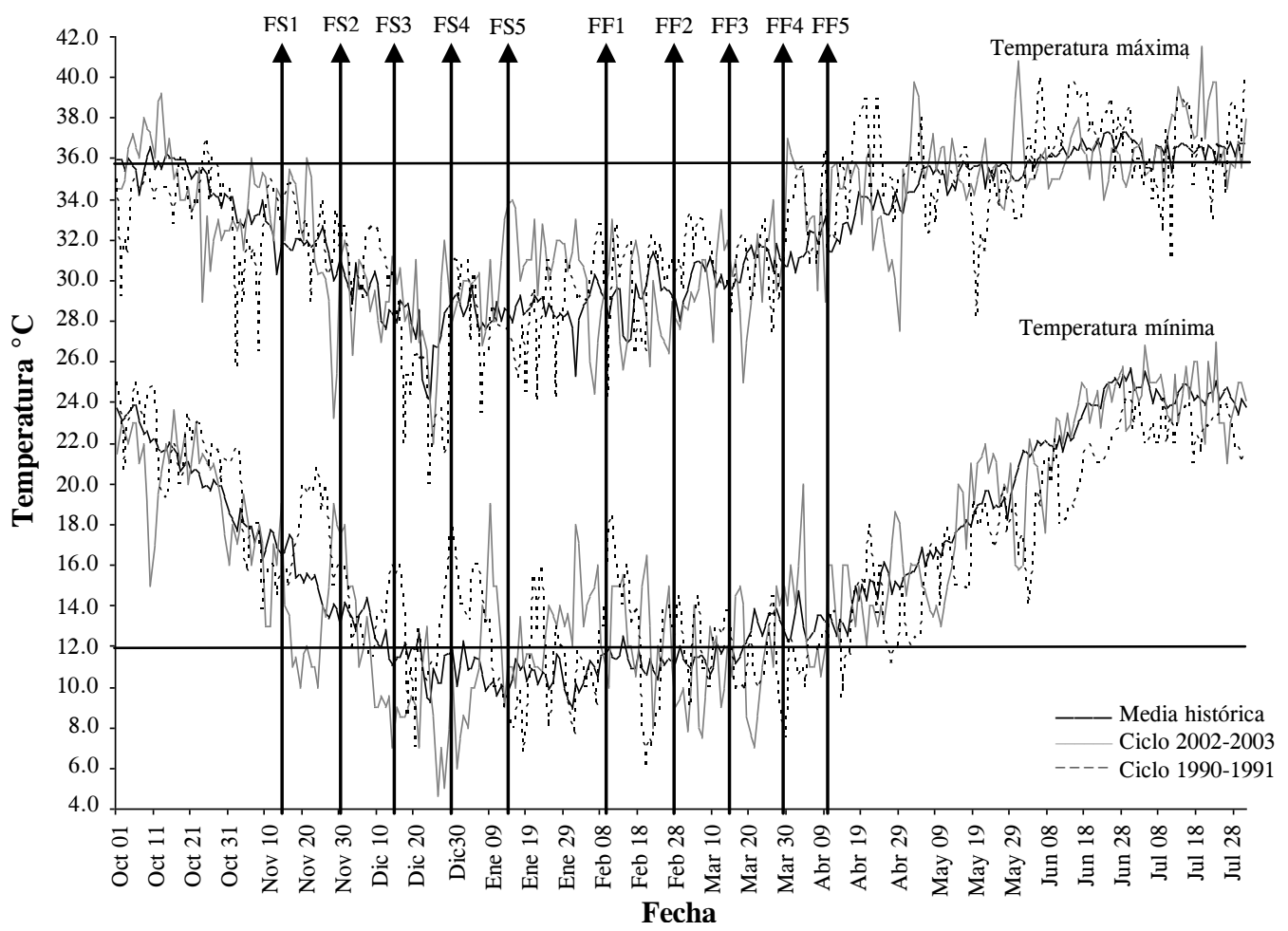

Figura 2. Distribución diaria de las temperaturas máxima y mínima en los ciclos 1990-1991, 2002-2003 y el promedio histórico (1981-2003), en Culiacán, Sin. Las líneas horizontales representan las temperaturas umbrales. FS = Fecha de siembra; FF = Floración femenina.

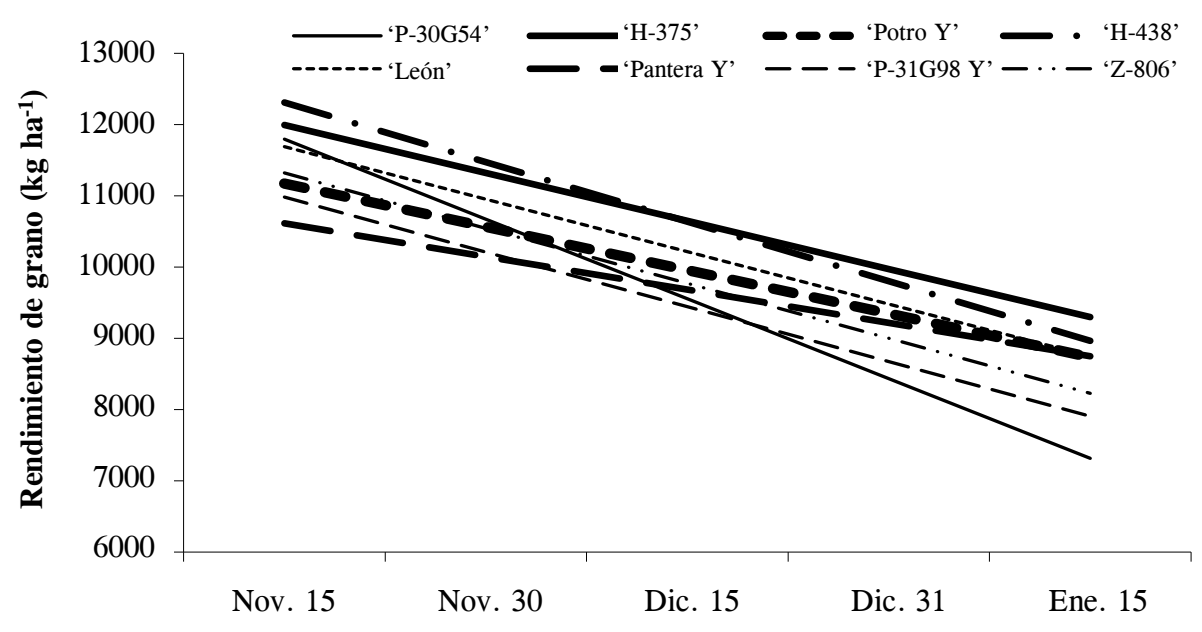

Figura 3. Rendimiento de grano ajustado $\left(\mathrm{kg} \mathrm{ha}^{-1}\right)$ en función del híbrido y la fecha siembra. Culiacán, Sin. Ciclo Otoño-Invierno 2002-2003. 
Los híbridos de grano blanco 'H-375' y 'H-438' mostraron el rendimiento de grano más alto en promedio de las cinco fechas de siembra, con 10648 y $10638 \mathrm{~kg} \mathrm{ha}^{-1}$ respectivamente, mayor porcentaje de acame de raíz y fueron ligeramente más tardíos; de éstos, ' $\mathrm{H}-375$ ' sobresale por su tolerancia a roya y mejor aspecto de mazorca. Ninguno de los híbridos de grano amarillo superó al 'H-375', lo que confirma que los híbridos de maíz de grano amarillo disponibles en el mercado no superan en rendimiento a los mejores de grano blanco (Ramírez et al., 2004); el híbrido 'Potro Y' tuvo el rendimiento más alto y el peor fue 'P-31G98 Y' (Cuadro 3).

La ausencia de paralelismo en el rendimiento de grano de los híbridos a través de las fechas de siembra demuestra el efecto de la interacción (Figura 3). Si bien todos los híbridos produjeron su máximo rendimiento en la siembra de noviembre 15, 'P-30G54', el más sembrado en Sinaloa, presentó la mayor reducción al cambiar la fecha de siembra (1120 kg ha-1 por cada $15 \mathrm{~d}$ de retraso respecto a noviembre 15) (Cuadro 4), lo que explica el que los productores hayan adelantado la fecha de siembra. Los híbridos 'H-438', 'P-31G98' y 'Z-806' también mostraron reducciones considerables en rendimiento de grano $\left(837,770\right.$ y $773 \mathrm{~kg} \mathrm{ha}^{-1}$, respectivamente). El híbrido 'Pantera $\mathrm{Y}^{\prime}$ ' fue el de menor reducción (467 kg $\mathrm{ha}^{-1}$ ), pero su media de rendimiento en la primera fecha de siembra fue la más baja de los ocho híbridos.

La fecha de siembra en la que se produjo el rendimiento de grano más alto en el ciclo agrícola 2002-2003 fue noviembre $15,32 \mathrm{~d}$ antes que la de diciembre 17 informada por Medina (1993; Opus cit.) Estas diferencias se atribuyen, en parte, a las variaciones ambientales entre los dos ciclos agrícolas; pero en mayor grado a la dinámica en prácticas culturales, a las nuevas variedades sembradas (cruzas trilineales y simples), y a la estrategia aplicada en el manejo del germoplasma y la selección de variedades mejoradas durante el periodo de 1990-1991 a 2002-2003. Al respecto, en el ciclo agrícola de PV la selección de genotipos se hace en regiones subtropicales como Tlajomulco, Jal., donde el INIFAP y las empresas multinacionales Monsanto y Pioneer tienen sus campos experimentales sedes, y en el OI en Sinaloa; por tanto, las variedades poseen ahora más germoplasma de origen subtropical que tropical e incluso algunas empresas lo combinan con germoplasma de clima templado, debido a que éste aporta alelos para precocidad, reducción en porte de planta, acame e incremento en índice de cosecha (Oyervides et al., 1985; Abadassi y Hervé, 2000), lo cual ha influido en que las variedades se adapten simultáneamente a ambos ciclos (PV y OI), como es el caso del híbrido 'H-375' (Ramírez et al., 2005). Este enfoque también ha permitido que estos genotipos toleren más las temperaturas mínimas que ocurren durante el invierno que los genotipos derivados de la raza Tuxpeño (Ruiz et al., 1998), usada en variedades tropicales como ' $\mathrm{V}-424$ ' y 'V455’ (Gámez et al., 1996; CIMMYT, 1998).

El periodo oficial de siembra para maíz de OI que señala la SAGARPA (2003) para Culiacán es del primero de noviembre al 15 de diciembre. Si el periodo de siembra se ubicara entre octubre 10 y noviembre 30 los productores que siembren antes del primero de noviembre no tendrían aseguramiento. En cambio, los que siembran al final (diciembre 15) estarían perdiendo $748 \mathrm{~kg} \mathrm{ha}^{-1}$; si

Cuadro 3. Rendimiento de grano y características agronómicas de ocho híbridos de maíz (promedio de cinco fechas de siembra). Culiacán, Sin. Ciclo Otoño-Invierno 2002-2003.

\begin{tabular}{|c|c|c|c|c|c|c|c|c|c|c|}
\hline \multirow[t]{2}{*}{ Híbrido } & \multirow{2}{*}{$\begin{array}{l}\text { Rendimiento } \\
\left(\mathrm{kg} \mathrm{ha}^{-1}\right)\end{array}$} & \multirow{2}{*}{$\begin{array}{l}\text { Mazorcas } \\
\text { por } \\
\text { planta }\end{array}$} & \multicolumn{2}{|c|}{ Floración (d) } & \multicolumn{2}{|c|}{ Altura $(\mathrm{cm})$} & \multicolumn{2}{|c|}{ Acame (\%) } & \multirow{2}{*}{$\begin{array}{l}\text { Calif. } \\
\text { roya }^{\dagger}\end{array}$} & \multirow{2}{*}{$\begin{array}{c}\text { Aspecto de } \\
\text { mazorca }^{\ddagger}\end{array}$} \\
\hline & & & $\hat{0}$ & 오 & Planta & Mazorca & Raíz & Tallo & & \\
\hline 'H-375' & $10648 \mathrm{a}$ & $0.99 \mathrm{bc}$ & $92 \mathrm{~b}$ & $91 \mathrm{~b}$ & 222 cde & $111 \mathrm{~d}$ & $3.52 \mathrm{a}$ & 0.44 & $8.3 \mathrm{a}$ & $8.9 \mathrm{a}$ \\
\hline 'H-438' & 10638 a & $0.99 \mathrm{bc}$ & $93 \mathrm{a}$ & $93 \mathrm{a}$ & $220 \mathrm{de}$ & $121 \mathrm{ab}$ & $3.71 \mathrm{a}$ & 0.23 & $7.7 \mathrm{c}$ & $8.8 \mathrm{ab}$ \\
\hline ‘León’' & $10218 \mathrm{~b}$ & $0.96 \mathrm{c}$ & $89 \mathrm{c}$ & $89 \mathrm{c}$ & 227 bcde & $125 \mathrm{a}$ & $2.31 \mathrm{bc}$ & 0.59 & $8.0 \mathrm{ab}$ & $8.3 \mathrm{dc}$ \\
\hline 'Potro Y' & 9954 bc & $1.00 \mathrm{~b}$ & $86 \mathrm{~d}$ & $86 \mathrm{~d}$ & $233 a b c$ & $121 \mathrm{ab}$ & $1.68 \mathrm{~cd}$ & 0.30 & $8.2 \mathrm{a}$ & $8.4 \mathrm{c}$ \\
\hline 'Z-806' & $9773 \mathrm{~cd}$ & $1.02 \mathrm{~b}$ & $86 \mathrm{~d}$ & $86 \mathrm{~d}$ & $234 \mathrm{ab}$ & $125 \mathrm{a}$ & $3.58 \mathrm{a}$ & 0.43 & $7.9 \mathrm{bc}$ & $8.2 \mathrm{dc}$ \\
\hline 'Pantera Y' & $9682 \mathrm{~cd}$ & $0.96 \mathrm{c}$ & $88 \mathrm{c}$ & $88 \mathrm{c}$ & $218 \mathrm{e}$ & $120 \mathrm{abc}$ & $2.47 \mathrm{~b}$ & 0.17 & $8.3 \mathrm{a}$ & $8.5 \mathrm{bc}$ \\
\hline 'P-30G54' & $9554 \mathrm{~cd}$ & $0.99 \mathrm{bc}$ & $89 \mathrm{c}$ & $89 \mathrm{c}$ & $241 \mathrm{a}$ & $117 \mathrm{bcd}$ & $1.20 \mathrm{~d}$ & 0.26 & $7.9 \mathrm{ab}$ & $8.0 \mathrm{~d}$ \\
\hline 'P-31G98 Y' & $9443 \mathrm{~d}$ & $1.11 \mathrm{a}$ & $86 \mathrm{~d}$ & $86 \mathrm{~d}$ & $230 \mathrm{abcd}$ & $113 \mathrm{~cd}$ & $1.15 \mathrm{~d}$ & 0.09 & $7.3 \mathrm{~d}$ & $7.1 \mathrm{e}$ \\
\hline $\operatorname{DMS}_{(0.05)}$ & 420 & 0.04 & 0.77 & 0.81 & 11.4 & 7.1 & 0.76 & NS & 0.5 & 0.3 \\
\hline
\end{tabular}


Cuadro 4. Regresión lineal, coeficiente de determinación y de correlación entre rendimiento del híbrido y fecha de siembra. Culiacán, Sin. Ciclo Otoño-Invierno 2002-2003.

\begin{tabular}{lcccc}
\hline Híbrido & $\begin{array}{c}\text { Intercepto } \\
\left(\mathrm{kg} \mathrm{ha}^{-1}\right)\end{array}$ & $\begin{array}{c}\text { Coef. Regresión } \\
\left(\mathrm{kg} \mathrm{ha}^{-1}\right)\end{array}$ & $\begin{array}{c}\text { Coef. Determinación } \\
\left(\mathrm{R}^{2}\right)\end{array}$ & $\begin{array}{c}\text { Coef. Correlación } \\
(\mathrm{r})\end{array}$ \\
\hline 'P-30G54' & 12913 & $-1120^{* *}$ & 0.99 & $0.99^{* *}$ \\
'H-375' & 12670 & $-674^{* *}$ & 0.91 & $0.96^{* *}$ \\
'Potro Y' & 11782 & $-609^{*}$ & 0.91 & $0.95^{*}$ \\
'H-438' & 13148 & $-837^{*}$ & 0.85 & $0.92^{*}$ \\
'León' & 12424 & $-736^{* *}$ & 0.93 & $0.96^{* *}$ \\
'Pantera Y' & 11083 & $-467^{* *}$ & 0.96 & $0.98^{* *}$ \\
'P-31G98' & 11752 & $-770^{* *}$ & 0.92 & $0.96^{* *}$ \\
'Z-806' & 12092 & -773 & 0.75 & 0.86
\end{tabular}

${ }^{*, * *}$ Valor significativo a 0.05 y 0.01 de probabilidad de error, respectivamente.

se considera el efecto de interacción genotipo $\mathrm{x}$ ambiente, en híbridos como 'P-30G54' la pérdida sería de $1120 \mathrm{~kg}$ $\mathrm{ha}^{-1}$ (Cuadro 4). Por tanto, se requiere explorar fechas de siembra desde el primero de octubre hasta diciembre 15 con los híbridos comerciales en uso. Además, se sugiere incluir híbridos tropicales de ciclo tardío como alternativa de siembras tardías, debido a que toleran mejor las altas temperaturas y tendrían un periodo de llenado de grano más largo que los híbridos de ciclo precoz e intermedio. Otra opción es determinar las temperaturas cardinales de los híbridos comerciales (Ruiz et al., 2002), y sembrarlos en fechas que cubran su requerimiento térmico a lo largo del ciclo agrícola.

\section{CONCLUSIONES}

Para el ciclo de otoño-invierno la mejor fecha de siembra para rendimiento de grano en el Valle de Culiacán, Sinaloa, en el 15 de noviembre. Los híbridos de grano blanco ' $\mathrm{H}-375$ ' y 'H-438', en promedio de las cinco fechas de siembra, presentan el mayor rendimiento, y 'P-31G98 Y' de grano amarillo el rendimiento más bajo. La temperatura máxima diaria es la de mayor influencia en los cambios en rendimiento asociados con la fecha de siembra.

\section{BIBLIOGRAFÍA}

Abadassi J, Y Hervé (2000) Introgression of temperate germplasm to improve an elite tropical maize population. Euphytica 113:125-133.

Benacchio S S (1982) Algunas Exigencias Agroecológicas en 58 Especies de Cultivo con Potencial de Producción en el Trópico Americano. FONAIAP-Centro Nal. de Inv. Agropec. Ministerio de Agricultura y Cría. Maracay, Venezuela. 202 p.

Barrios A, A, A Turrent F, J I Cortés F, C A Ortiz S, N O Gómez M, A Martínez $\mathbf{G}$ (2004) Interacción genotipo x prácticas de manejo en híbridos de maíz. Efecto sobre el diseño de recomendaciones. Rev. Fitotec. Mex. 4:399-408.
CIMMYT (1998) A Complete Listing of Improved Maize Germplasm from CIMMYT Maize Program Special Report. Mexico, D. F. $\mathrm{pp}: 15-16$.

Cross H Z, M S Zuber (1972) Prediction in flowering in maize based on different methods of estimating thermal units. Agron. J. 64:351355.

Eskridge K M, E J Stevens (1987) Growth curve analysis of temperature dependent phenology models. Agron. J. 79:291-297.

Francis C A (1972) Photoperiod sensitivity and adaptation in maize. 27th Annual Corn \& Sorghum Research Conference. Chicago, ILL. USA. pp:119-131.

Gámez V A J, M A Ávila P, H H Ángeles A, C Díaz H, H Ramírez V, A. Alejo J, A D Terrón I (1996) Híbridos y Variedades de Maíz Liberados por el INIFAP hasta 1996. Publicación Especial No. 6. INIFAP. México, D. F. 103 p.

González A I J, J A Ruiz C, J Anguiano C, I Vizcaino V (2005) Recursos Edafo-Climáticos para la Planeación del Sector Productivo en el Estado de Nayarit. Libro Técnico Núm. 1 INIFAP-CIRPACCampo Exptal. Santiago Ixcuintla. Santiago Ixcuintla, Nayarit.171 p.

Kucharik, C J (2006) A multidecadal trend of earlier corn planting in Central USA. Agron. J. 98:1544-1550.

Martínez G A (1996) Diseños Experimentales: Métodos y Elementos de Teoría. Ed. Trillas. México. pp:664-694.

Ojeda B W, E Sifuentes I, H Unland W (2006) Programación integral del riego en maíz en el norte de Sinaloa, México. Agrociencia 40:13-25.

Oyervides G M, A R Hallauer, H Cortez M (1985) Evaluation of improved maize populations in Mexico and U.S. Corn Belt. Crop Sci. 25:115-120.

Ramírez D J L, M Chuela B, L Soltero D, J Franco M, A Morfín V, V A Vidal M, H L Vallejo D, F Caballero H, H Delgado $M, ~ R$ Valdivia B y J Ron P (2004) Patrón heterótico de maíz amarrillo para la región Centro-Occidente de México. Rev. Fitotec. Mex. 27:13-17.

Ramírez D J L, M Chuela B, V A Vidal M, J J Wong P, H Córdova O, L Soltero D, J Franco M, H L Vallejo D, A Arregui E, A Morfín V, F Caballero H, H Delgado M, J Ron P, J J Sánchez G, G Vázquez C (2005) H-375, Híbrido de Maíz de Grano Blanco para Riego y Buen Temporal en la Región Centro Occidente, y Riego en el Noroeste de México. Folleto Técnico No. 1. Campo Experimental Centro-Altos de Jalisco. CIRPAC-INIFAP. Tepatitlán de Morelos, Jal. 28 p.

Ruiz C J A, J J Sánchez G, M Goodman (1998) Base temperature and heat unit requirement of 49 Mexican maize races. Maydica 43:277282. 
Ruiz C J A, H E Flores L, J L Ramírez D, D R González E (2002) Temperaturas cardinales y duración del ciclo de madurez del híbrido de maíz H-311 en condiciones de temporal. Agrociencia 36:569577.

Ruiz C J A, I J González A, J Anguiano C, I Vizcaíno V, D Ibarra C, J Alcalá G, S Espinoza V y H E Flores L (2003) Estadísticas Climatológicas Básicas para el Estado de Jalisco (Período 19612000). Libro Técnico Núm. 1. INIFAP-CIRPAC-C.E. Centro de Jalisco. Tlajomulco de Zúñiga, Jalisco. 281 p.

SAGARPA (2003) Boletín de Variedades Recomendadas de los Principales Cultivos con Indicaciones para las Épocas de Siembra y Cosecha Ciclo Otoño-Invierno 2003/2004. Dirección de Vinculación y Desarrollo Tecnológico. SAGARPA. México, D. F.

SAGARPA (2007) Avance de siembras y cosechas. Riego + Temporal. Disponible en: http://www.siap.sagarpa.gob.mx/ar_comfichedos .html (Abril de 2007).
SAS Institute (1996) SAS Software Release V 6.12, SAS Institute, Inc. Cary, N. C. $830 \mathrm{p}$

Shaw R H (1975) Growing degree units for corn in the North Central Region. North Central Region Res. Pub. Num. 229. Iowa State Univ. IWRBBR 581:793-808.

Singh P M, J R Gilley, W E Splinter (1976) Temperature thresholds for corn growth in a controlled environment. Trans. ASAE 19:1152-1155.

Snedecor G W, W G Cochran (1974) Métodos Estadísticos. 2a Imp. Ed. CECSA. México, D. F. pp:402-405.

Wiatrak, P J, D L Wright, J J Morois, R Sprenkel (2004) Corn hybrids for late planting in the Southeast. Agron. J. 96:1118-1124.

Wiatrak, P J, D L Wright, J J Morois, D Wilson (2005) Influence of planting date on aflotoxin accumulation in $\mathrm{Bt}$, non-Bt, and tropical non-Bt hybrids. Agron. J. 97:440-445. 